\title{
Factores predictores de recurrencia post ablación de fibrilación auricular
}

\author{
Mauricio Moreno ${ }^{1,2}$, Rodulfo Oyarzún ${ }^{1,2}$, Carlos Piedra ${ }^{2}$, Marcelo Orellana $^{2}$, Marianella Seguel. \\ 1 Unidad de Arritmias, Clínica Dávila. \\ 2 Laboratorio de Electrofisiología, Instituto Nacional del Tórax.
}

Introducción: Los resultados de la ablación de fibrilación auricular (FA) pueden ser afectados por diversas variables entre los cuales se cuenta el tipo de FA y la presencia de cardiopatía estructural.

Objetivo: El objetivo de este estudio es determinar qué factores se relacionan con la recurrencia de esta arritmia después de la ablación de FA.

Métodos: Los criterios de inclusión para ablación de FA fueron pacientes con FA sintomática refractaria a terapia antiarrítmica. El procedimiento consistió en el aislamiento eléctrico de las venas pulmonares, para lo cual se realizaron líneas de ablación alrededor de las VP a nivel antral y/u ostial. Se utilizó un sistema de navegación 3D: CARTO 3 o EnSITE NavX. Los pacientes fueron controlados clínicamente y con holter de arritmia para evaluar recurrencia de FA hasta 1 año post procedimiento. Se analizaron las siguientes variables para establecer su relación con el éxito del procedimiento: edad, sexo, tipo de FA, tiempo de evolución de la FA antes del procedimiento y tamaño de la aurícula izquierda.

Resultados: Se incluyeron 70 pacientes con ablación de fibrilación auricular. En el seguimiento alejado se observó recurrencia en 17 pacientes (24,3\%). De 11 pacientes de sexo femenino ninguna presentó recurrencia (p 0.055). La edad promedio de los pacientes con recurrencia fue $59.8 \pm 8.6$ años versus sin recurrencia $49.2 \pm 12.8$ años ( $\mathrm{p} 0.002)$. De 9 pacientes con FA persistente, $2(22.2 \%)$ presentaron recurrencia y 7 (77.7\%) no tuvieron recurrencia ( $\mathrm{p} \mathrm{NS}$ ). El tiempo de evolución promedio de la FA antes de la ablación fue $72.3 \pm 68.1$ meses en los recurrentes y $49.61 \pm 46.8$ meses en los no recurrentes (p 0.175). En los pacientes con recurrencia, el promedio de tamaño de la AI fue $41 \pm 4.7 \mathrm{~mm}$ y en los sin recurrencia fue $38.4 \pm 6.1 \mathrm{~mm}$ (p 0.133 ).

Conclusión: La ablación de FA es una terapia segura y efectiva para evitar las recurrencias de esta arritmia. La mayor edad es un predictor de mayor recurrencia post ablación de FA.

\section{Correspondencia:}

Dr. Mauricio Moreno

Clínica Dávila

E-mail: mauricio.moreno@clinicadavila.cl 


\section{Predictors of recurrence of atrial fibrillation after successful ablation therapy}

Background: Success in ablation for atrial fibrillation (AF) is affected by different conditions, such as type of $\mathrm{AF}$ and the presence of structural heart disease.

Aim: to determine factors associated to recurrence of AF after successful ablation therapy.

Method: Patients with AF unresponsive to pharmacologic treatment were included. Pulmonary veins isolation was performed by ablation lines around pulmonary veins at the antral or ostial levels. A 3-D navigation system (Carto 3 or Onsite NavX) was employed. Patients underwent clinical and holter monitoring up to 1 year post ablation in order to determine de recurrence of AF. Age, gender, type of $\mathrm{AF}$, duration of $\mathrm{AF}$ prior to ablation and left atrial size were explored as possible predictors of AF recurrence.

Results: 70 patients were included in the study. Re-

\section{Introducción:}

La fibrilación auricular (FA) es la arritmia sostenida más frecuente y determina un aumento de la morbimortalidad de los pacientes que la padecen. Se asocia a alteraciones hemodinámicas, alteración de la calidad de vida y alto riesgo de tromboembolia. Además del tratamiento anticoagulante el tratamiento de la FA puede incluir la restauración del ritmo sinusal o permitir que persista la FA con control de la frecuencia cardíaca.

Para mantener el ritmo sinusal se pueden usar antiarrítmicos, aunque tienen una baja eficacia y a menudo se asocian a efectos adversos, o se puede realizar ablación de la FA, que es una terapia potencialmente curativa para esta arritmia ${ }^{1}$.

Como en la mayoría de los pacientes la actividad ectópica que origina y/o mantiene la FA proviene de las venas pulmonares (VP), el objetivo principal de la ablación de FA es el aislamiento o desconexión eléctrica de las $\mathrm{VP}{ }^{1}$. La evolución de esta terapia también ha incorporado la ablación de sustratos auriculares que pueden favorecer la $\mathrm{FA}^{2}$. Hay diversos estudios que han evaluado el impacto de las variables clínicas y los factores relacionados con el procedimiento sobre los resultados de la ablación de FA (3-5). El objetivo de este estudio es analizar las variables clínicas que pueden afectar la recurrencia de un grupo de pacientes sometidos a ablación de FA. currence of $\mathrm{AF}$ occurred in 17 (24.3\%), none of them women $(\mathrm{n}=11, \mathrm{p}=0.055)$. Mean age in patients with $\mathrm{AF}$ recurrence was $59.8 \pm 8.6$ years compared to $49.2 \pm 12.8$ in those free of AF recurrence ( $\mathrm{p}=0.002)$. Out of $9 \mathrm{pa}-$ tients with persistent AF, 2 (22.2\%) had recurrence, and 7 (77.7\%) did not; this difference was no significant. Duration of $\mathrm{AF}$ prior to ablation was $72.3 \pm 68.1 \mathrm{vs} 49.61 \pm$ 46.8 months in patients with compared to patients without AF recurrence, $(\mathrm{p}=0.175)$. Left atrial dimension was $41 \pm$ $4.7 \mathrm{~mm}$ in patients with $\mathrm{AF}$ recurrence compared to 38.4 $\pm 6.1 \mathrm{~mm}$ in those without recurrence $(\mathrm{p}=0.133)$

Conclusion: Ablation of AF is safe and effective to prevent $\mathrm{AF}$ recurrence. Increasing age predicts a higher rate of recurrence

Keywords: atrial fibrillation, recurrence, ablation, predictive factors

\section{Métodos:}

\section{Características de los pacientes}

Se incluyeron 70 pacientes consecutivos sometidos a ablación de FA cuya indicación fue FA paroxística sintomática recurrente a pesar de tratamiento antiarrítmico y FA persistente sintomática sin cardiopatía estructural significativa. Se excluyeron los pacientes con enfermedad cardíaca estructural avanzada. En la tabla 1 se describen las características más relevantes de los pacientes.

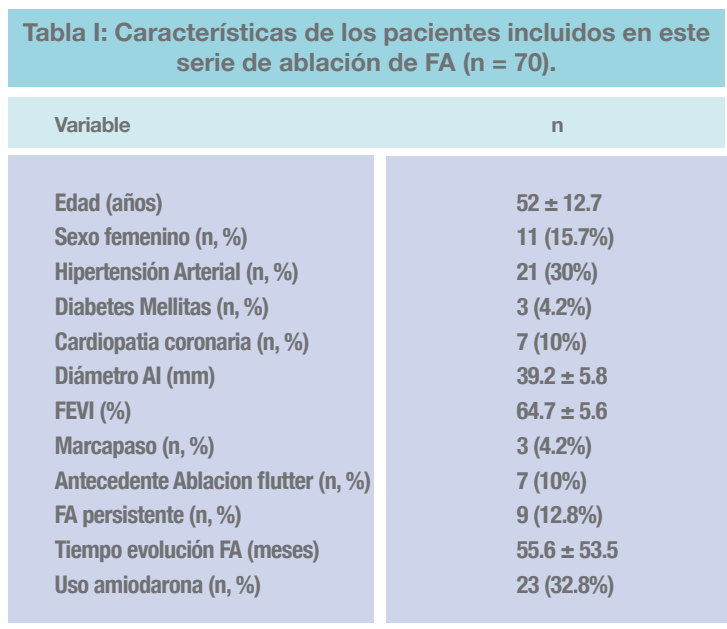

$F E V I=$ fraccion de eyeccion del ventrículo izquierdo 


\section{Estudio electrofisiológico}

El procedimiento de ablación de FA ha sido descrito previamente $^{6}$. Se realizó bajo sedación profunda con infusión continua de propofol. Se realizaron 3 punciones venosas femorales derechas para avanzar catéteres diagnósticos decapolar al seno coronario y catéter lasso (Biosense Webster) para registrar la actividad eléctrica de las VP, y catéter de ablación irrigado abierto Sprinkler (Medtronic) o NaviStar Thermocool (Biosense Webster). Para avanzar los catéteres a la aurícula izquierda (AI) se efectuó una punción transeptal guiada por radioscopía. Después del acceso transeptal se administró heparina endovenosa para mantener un tiempo de coagulación activado alrededor de 300 segundos, con controles cada 20 a 30 minutos. En algunos casos se efectuó angiografía de las VP. Para registrar las señales eléctricas intracardíacas se utilizó un polígrafo EP-TRACER (CardioTek). En la mayoría de los casos se construyó un mapa anatómico tridimensional de aurícula izquierda y VP con el sistema de navegación EnSITE-NAVx (St Jude Medical) o CARTO 3 (Biosense Webster). Generalmente este mapa resultó de la fusión entre la información eléctrica de un catéter (de ablación o lasso) y una imagen de la AI y VP por tomografía multicorte realizada previamente.

\section{Ablación}

Los parámetros de ablación con radiofrecuencia (RF) fueron temperatura $45^{\circ} \mathrm{C}$ y potencia 30 watts. Los potenciales dentro de las VP fueron registrados con el catéter lasso. El aislamiento de cada VP consistió en el bloqueo eléctrico bidireccional a la AI, lo cual se estableció cuando desaparecieron o se disociaron los potenciales de VP y no hubo conducción a la AI al estimular dentro de la VP.

La estrategia de ablación consistió en el aislamiento eléctrico empírico de todas las VP que presentaban conducción eléctrica y ocasionalmente de otras estructuras, como la vena cava superior (VCS). En otros casos la estrategia de ablación fue focal, es decir, se aisló sólo a la(s) VP con mayor probabilidad de ser culpable del inicio de la FA, para lo cual se eligió la VP donde se originaban las ectopias auriculares espontáneas o inducidas con adenosina y/o isoproterenol. La aplicación RF se efectuó a nivel ostial o a nivel antral de las VP. En la ablación ostial las aplicaciones fueron en el ostium de la VP involucrando todo su perímetro o parte de él (segmentaria). En la ablación antral se aplicó RF en la unión venoatrial de VP ipsilaterales, creando líneas circunferenciales alrededor de ellas. Estas estrategias de ablación habitualmente fueron guiadas a través de un mapa anatómico tridimensional (ver fig. 1 y 2). Siempre el objetivo fue la desconexión eléctrica de las VP. Si la ablación antral no lograba desconectar eléctricamente la VP, se realizaron aplicaciones adicionales de RF ostiales hasta cumplir este objetivo. Aquellas VP que no mostraron conducción eléctrica, no fueron ablacionadas.
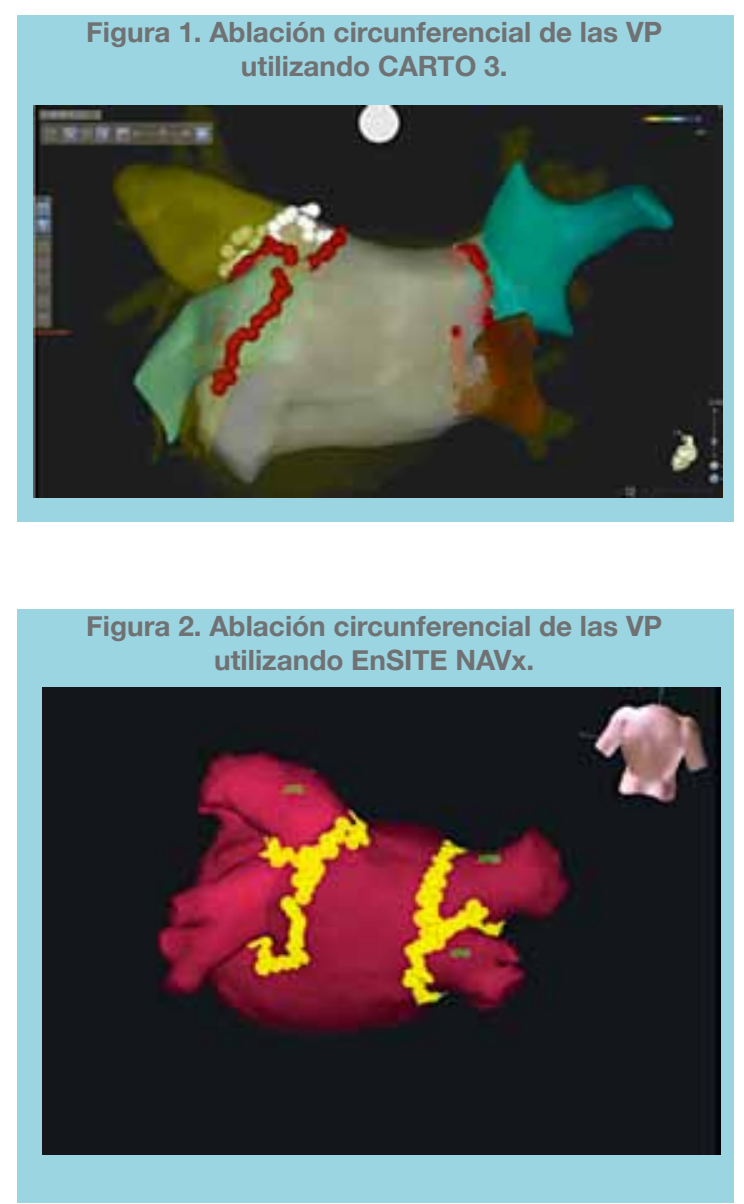

Posterior al procedimiento, los pacientes quedaron en la unidad coronaria y a las 6 horas se administró clexane subcutáneo en dosis 50\% de la terapéutica. Al día siguiente del procedimiento, se realizó un ecocardiograma transtorácico de control. Se indicó un antiarrítmico por 1 a 3 meses y al tercer mes se evaluó la suspensión del anticoagulante según el riesgo tromboembólico del paciente. Para evaluar la recurrencia de FA los pacientes fueron seguidos clínicamente y con holter de arritmias de 24 horas dentro de los 3 meses pos ablación, al sexto mes y al año. Se definió como recurrencia de FA post ablación cuando el paciente presentó un episodio clínico de FA con o sin documentación de la arritmia, o un episodio de FA registrado en el holter de arritmias. Las recurrencias observadas durante los primeros 3 meses post ablación no fueron consideradas para el análisis de los resultados (período de ventana). Las variables clínicas más relevantes fueron correlacionadas con la recurrencia de FA después de un primer procedimiento de ablación de FA. 


\section{Análisis estadístico}

Las variables continuas se expresan con promedio y desviación estándar. Para el estudio se efectuó un análisis univariado, donde se utilizó chi cuadrado o test exacto de Fisher cuando estaba indicado para analizar las variables dicotómicas. Para las variables continuas, se utilizó t test. Se consideró significativo un valor de p de 0.05 .

\section{Resultados:}

Después de una primera intervención de ablación de FA se observó recurrencia de FA en 24 (34.3\%) de los 70 pacientes. De estos 24 pacientes con recurrencia, 11 (45.8\%) fueron reintervenidos, y $13(54.2 \%)$ recibieron tratamiento médico para control de ritmo o control de frecuencia cardíaca. De los 11 pacientes sometidos a una segunda ablación, 5 (45.4\%) presentaron nueva recurrencia de la FA y $6(54.6 \%)$ permanecieron libre de FA. De los 5 pacientes con recurrencia de FA post segunda ablación, uno fue sometido a una tercera ablación y permaneció libre de FA, posteriormente. En resumen, durante un seguimiento promedio de $20 \pm 12$ meses con una tasa de reintervención de $17.1 \%$, se observó recurrencia de FA en 17 (24.2\%) de 70 pacientes.

En $52(74.3 \%)$ pacientes se utilizó EnSITE NAVx, en $7(10 \%)$ pacientes CARTO 3 y en 11 (15.7\%) pacientes no se utilizó un sistema de navegación 3D. Respecto a la primera intervención, fue ablación focal en 24 (34.3\%) pacientes y ablación de todas las VP en 46 (65.7\%) pacientes. De los 11 pacientes sometidos a una segunda intervención fue focal en 3 (27.3\%) pacientes y de todas las VPen 8 (72.7\%) pacientes. En 3 VP no se logró su aislamiento eléctrico. En los 20 (28.5\%) pacientes que se realizó ablación de la VCS se alcanzó su desconexión eléctrica. No se observaron diferencias estadísticamente significativas entre estas estrategias de ablación y recurrencia de FA.

En la tabla 2 se muestran las variables clínicas mas relevantes y su relación con la recurrencia de FA post ablación. Una edad mayor se asoció en forma significativa a una mayor recurrencia de FA ( $\mathrm{p}=0.002)$, mientras que el sexo femenino mostró una tendencia a menor recurrencia.

\begin{tabular}{|c|c|c|c|}
\hline \multicolumn{4}{|c|}{$\begin{array}{l}\text { Tabla II: Comparación de variables clínicas con la } \\
\text { recurrencia de FA post ablación. }\end{array}$} \\
\hline \multirow[t]{2}{*}{ Variable } & \multicolumn{2}{|c|}{ Recurrencia } & \multirow{2}{*}{$\mathbf{P}$} \\
\hline & $\mathrm{Si}$ & No & \\
\hline Sexo femenino (11) & $11(100 \%)$ & 0 & 0.055 \\
\hline Edad (años) & $49.2 \pm 12.8$ & $59.8 \pm 8.6$ & 0.002 \\
\hline FA persistente (9) & $7(77.7 \%)$ & $2(22.2 \%)$ & 1.0 \\
\hline HTA (21) & $13(61.9 \%)$ & $8(38 \%)$ & 0.135 \\
\hline Tiempo FA (meses) & $49.61 \pm 46.8$ & $72.3 \pm 68.1$ & 0.175 \\
\hline Diámetro $\mathrm{Al}(\mathrm{mm})$ & $38.4 \pm 6.1$ & $41 \pm 4.7$ & 0.133 \\
\hline
\end{tabular}

Se observó un caso estenosis de las VP izquierdas sintomáticas en un paciente que fue tratado exitosamente con angioplastía sin stent. Presentaron derrame pericárdico post ablación 2 pacientes, se manejaron con reversión de la anticoagulación y no fue necesario su drenaje.

\section{Discusión:}

El éxito de la ablación de FA, entendido como ausencia de FA sin necesidad de antiarrítmicos, varía entre 70 a $85 \%$. Estos resultados pueden disminuir cuando se incluyen pacientes con FA crónica, alteraciones estructurales cardíacas importantes y según la experiencia del centro 7 . ${ }^{8}$. En esta serie se observó una recurrencia de FA del $24 \%$, incluyendo los pacientes reintervenidos (17\%), lo cual es comparable con la información publicada.

En un reciente estudio se analizaron los predictores clínicos de recurrencia de FA después del aislamiento antral de las VP ${ }^{9}$. Los autores encontraron que la FA no paroxística, hipertensión arterial y el antecedente de falla a un antiarrítmico fueron predictores de mayor recurrencia de FA post ablación, mientras que la edad no fue un factor predictor de recurrencia. Hay importantes diferencias entre este estudio y la serie que reportamos. Incluyeron $37 \%$ pacientes con FA no paroxística versus $13 \%$ de FA persistente de nuestro estudio. Nuestra serie incluyó pacientes más jóvenes con edad promedio de 52 años, versus 58 años del estudio de Khaykin. La duración de la FA fue 7.5 años, versus 4.5 años de nuestro estudio. El antecedente de hipertensión arterial ocurrió en el $45 \%$ de los pacientes de la serie de Khaykin, mientras que sólo el $30 \%$ de los pacientes de esta serie tenía este antecedente. El tamaño de la AI fue más pequeño en nuestra serie, 39 versus $42 \mathrm{~mm}$. El $24 \%$ de los pacientes presentaban cardiopatía estructural significativa en la serie de Khaykin a diferencia del antecedente de cardiopatía coronaria del $10 \%$ de pacientes de esta serie. Todas estas diferencias orientan a que la serie que publicamos es un grupo de pacientes con menos daño estructural cardíaco y consecuentemente menor remodelado anatómico y electrofisiológico de la AI. Siendo poblaciones muy diferentes las variables relacionadas con la recurrencia de FA pueden diferir ampliamente.

Según nuestros resultados, a mayor edad se observa una mayor recurrencia de FA post ablación. Bhargava ${ }^{3}$ reportó los resultados de la ablación ostial de las VP según grupos etarios, para lo cual dividió los pacientes en $<50$ años, $51-60$ años y $>60$ años. Las tasas de recurrencias de FA fueron $15 \%, 16 \%$ y $18 \%$ respectivamente. Aunque no se alcanzó diferencia estadística, se observó una tendencia a aumentar la recurrencia a mayor 
edad. Es posible que los cambios estructurales (fibrosis) y electrofisiológicos que experimentan las aurículas con la edad expliquen que los resultados de la ablación sean peores. Estos datos sugieren que una aproximación más precoz del manejo de la FA con ablación puede determi- nar mejores resultados con esta terapia.

En conclusión, la ablación de FA es una terapia segura y efectiva para evitar las recurrencia de esta arritmia. Una mayor edad puede ser un predictor de mayor recurrencia post ablación de FA.

\section{Referencias:}

1. HAÏSSAGUERRE M, JAIMLIS P, SHAH DC, TAKAHASHI A, HOCINI M, QUINIOU G, et al. Spontaneous initiation of atrial fibrillation by ectopic beats originating in the pulmonary veins. N Engl J Med. 1998;339:659-666.

2. NADEMANEE K, MCKENZIE J, KOSAR E, SCHWAB M, SUNSANEEWITAYAKUL B, VASAVAKUL T, et al. A new approach for catheter ablation of atrial fibrillation: Mapping of the electrophysiologic substrate. J Am Coll Cardiol 2004; 43: $2044-2053$

3. BHARGAVA M, MARROUCHE NF, MARTIN DO, SCHWEIKERT RA, SALIBA W, SAAD EB, et al. Impact of age on the outcome of pulmonary vein isolation for atrial fibrillation using circular mapping technique and cooled-tip ablation catheter. J Cardiovasc Electrophysiol 2004; 15: 8 - 13.

4. KHAYKIN Y, MARROUCHE NF, SALIBA W, SCHWEIKERT R, BASH D, CHEN MS, et al. Pulmonary vein antrum isolation for treatment of atrial fibrillation in patients with valvular heart disease or prior open heart surgery. Heart Rhythm 2004; 1: 33 - 39.
5. CHEN MS, MARROUCHE NF, KHAYKIN Y, GILLINOV AM, WAZNI O, MARTIN DO, et al. Pulmonary vein isolation for the treatment of atrial fibrillation in patients with impaired systolic function. J Am Coll Cardiol 2004; 43: 1004 - 1009.

6. MORENO M, OYARZUN R, BONILLA C, TAPIA R, MARTÍNEZ E, LARREA R. Estrategias de aislamiento de las venas pulmonares durante la ablación de fibrilación auricular. Rev Chil Cardiol 2011; 31: 140 - 144.

7. JALIS P, CAUCHEMEZ B, MACLE L, DAOUD E, KHAIRY $\mathrm{P}$, SUBBIAH R, et al. Catheter ablation versus antiarrhythmic drugs for atrial fibrillation: the A4 study. Circulation. 2008; 118: $2498-2505$

8. CALKINS H, REYNOLDS MR, SPECTOR P, SONDHI M, XU Y, MARTINA, et al. Treatment of atrial fibrillation with antiarrhythmic drugs or radiofrequency ablation: two systematic literature reviews and meta-analyses. Circ Arrhythm Electrophysiol. 2009; 2: 349-361.

9. KHAYKIN Y, OOSTHUIZEN R, ZARNETT L, ESSEBAG V, PARKASH R, SEABROOK C, et al. Clinical predictors of arrhythmia recurrences following pulmonary vein antrum isolation for atrial fibrillation. J Cardiovasc Electrophysiol 2011; 22: 1206 $-1214$. 


\title{
Factores predictores de recurrencia post ablación de fibrilación auricular
}

\author{
Mauricio Moreno ${ }^{1,2}$, Rodulfo Oyarzún ${ }^{1,2}$, Carlos Piedra ${ }^{2}$, Marcelo Orellana $^{2}$, Marianella Seguel. \\ 1 Unidad de Arritmias, Clínica Dávila. \\ 2 Laboratorio de Electrofisiología, Instituto Nacional del Tórax.
}

Introducción: Los resultados de la ablación de fibrilación auricular (FA) pueden ser afectados por diversas variables entre los cuales se cuenta el tipo de FA y la presencia de cardiopatía estructural.

Objetivo: El objetivo de este estudio es determinar qué factores se relacionan con la recurrencia de esta arritmia después de la ablación de FA.

Métodos: Los criterios de inclusión para ablación de FA fueron pacientes con FA sintomática refractaria a terapia antiarrítmica. El procedimiento consistió en el aislamiento eléctrico de las venas pulmonares, para lo cual se realizaron líneas de ablación alrededor de las VP a nivel antral y/u ostial. Se utilizó un sistema de navegación 3D: CARTO 3 o EnSITE NavX. Los pacientes fueron controlados clínicamente y con holter de arritmia para evaluar recurrencia de FA hasta 1 año post procedimiento. Se analizaron las siguientes variables para establecer su relación con el éxito del procedimiento: edad, sexo, tipo de FA, tiempo de evolución de la FA antes del procedimiento y tamaño de la aurícula izquierda.

Resultados: Se incluyeron 70 pacientes con ablación de fibrilación auricular. En el seguimiento alejado se observó recurrencia en 17 pacientes (24,3\%). De 11 pacientes de sexo femenino ninguna presentó recurrencia (p 0.055). La edad promedio de los pacientes con recurrencia fue $59.8 \pm 8.6$ años versus sin recurrencia $49.2 \pm 12.8$ años ( $\mathrm{p} 0.002)$. De 9 pacientes con FA persistente, $2(22.2 \%)$ presentaron recurrencia y 7 (77.7\%) no tuvieron recurrencia ( $\mathrm{p} \mathrm{NS}$ ). El tiempo de evolución promedio de la FA antes de la ablación fue $72.3 \pm 68.1$ meses en los recurrentes y $49.61 \pm 46.8$ meses en los no recurrentes (p 0.175). En los pacientes con recurrencia, el promedio de tamaño de la AI fue $41 \pm 4.7 \mathrm{~mm}$ y en los sin recurrencia fue $38.4 \pm 6.1 \mathrm{~mm}$ (p 0.133 ).

Conclusión: La ablación de FA es una terapia segura y efectiva para evitar las recurrencias de esta arritmia. La mayor edad es un predictor de mayor recurrencia post ablación de FA.

\section{Correspondencia:}

Dr. Mauricio Moreno

Clínica Dávila

E-mail: mauricio.moreno@clinicadavila.cl 